\title{
EchoGéo
}

2 | 2007

Itinéraires et trajectoires des migrants

\section{Le détroit de Gibraltar}

Les limites d'un espace modèle de la lutte européenne contre les migrations irrégulières

Guillaume Le Boedec

\section{OpenEdition}

1 Journals

Édition électronique

URL : https://journals.openedition.org/echogeo/1488

DOI : 10.4000/echogeo.1488

ISSN : 1963-1197

Éditeur

Pôle de recherche pour l'organisation et la diffusion de l'information géographique (CNRS UMR 8586)

Référence électronique

Guillaume Le Boedec, "Le détroit de Gibraltar », EchoGéo [En ligne], 2 | 2007, mis en ligne le 22 février 2008, consulté le 31 juillet 2021. URL : http://journals.openedition.org/echogeo/1488 ; DOI : https:// doi.org/10.4000/echogeo. 1488

Ce document a été généré automatiquement le 31 juillet 2021.

EchoGéo est mis à disposition selon les termes de la licence Creative Commons Attribution - Pas d'Utilisation Commerciale - Pas de Modification 4.0 International (CC BY-NC-ND) 


\section{Le détroit de Gibraltar}

Les limites d'un espace modèle de la lutte européenne contre les migrations irrégulières

\section{Guillaume Le Boedec}

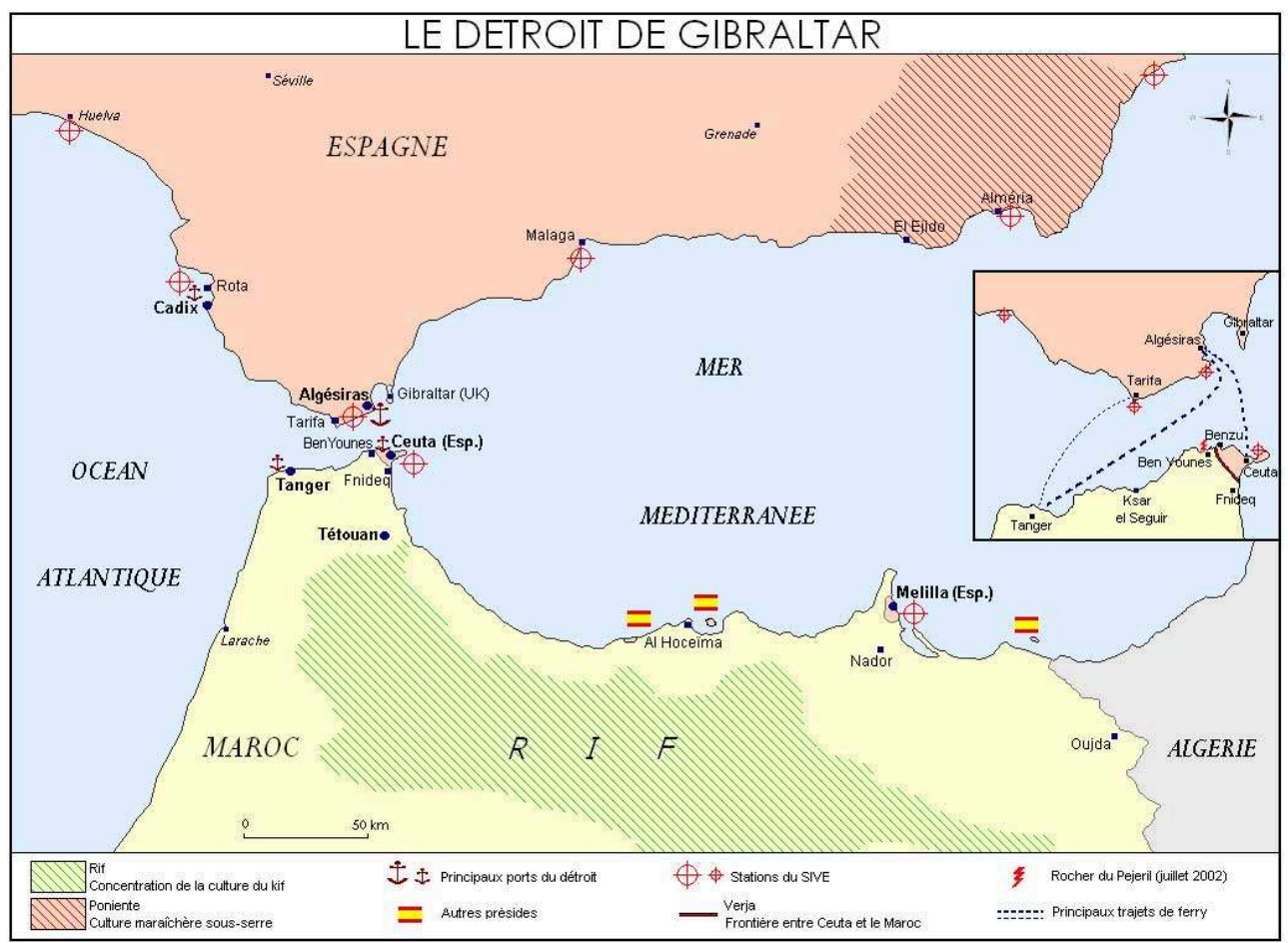

Conception: Guillaume Le Boedec

Né d'une déchirure terrestre que les mythes ont magnifiée, le détroit de Gibraltar a conservé à travers les siècles un statut de point nodal dans la géostratégie et la géopolitique mondiale. Porte océane entre Atlantique et Méditerranée, mais aussi porte transversale entre l'Afrique et l'Europe, le détroit capte et polarise les flux transméditerranéens. Avec seulement 13 kilomètres qui séparent les deux continents, il est le point de passage le plus étroit de la Méditerranée, mais aussi l'une des frontières les plus inégalitaires au monde. Alors que dans d'autres régions, la proximité favorise la 
convergence économique, l'écart de développement entre les deux rives ne cesse de croître : le PIB par habitant espagnol représente aujourd'hui quinze fois celui du Maroc alors qu'il n'était que quatre fois supérieur il y a trente ans.

2 Aujourd'hui, profitant de la proximité des deux rives, de nombreux trafics de contrebande et flux illégaux traversent l'étroit canal' ${ }^{1}$. Les narcotrafiquants, les contrebandiers et les clandestins ont remplacé les pirates et les corsaires d'hier. Les différents trafics, propres aux zones frontalières, ont contribué à transformer le détroit en une zone de non droit, un gigantesque marché tiers-mondiste, un entonnoir qui capte et redistribue sur l'ensemble de l'Europe les flux illégaux en provenance du Sud. Alimentés par le préside de Ceuta et par la colonie britannique de Gibraltar, ces trafics répondent à une logique d'offre et de demande :

le cannabis, le kif du Rif, contribue à la stabilité économique et sociale du nord du Maroc et alimente les réseaux mafieux de la costa del Sol qui le redistribuent à travers l'espace Schengen, la contrebande autour de Ceuta assure les revenus nécessaires à l'économie morose du préside et à la province de Tétouan, et enfin, les clandestins africains, en diminuant l'effectif de chômeurs de leur pays, constituent une soupape économique, mais aussi une main-d'œuvre utile et peu coûteuse pour la culture maraîchère sous serre d'Andalousie.

3 Le détroit de Gibraltar apparaît comme un point de passage obligé pour les filières de l'immigration clandestine. Au centre du phénomène pendant des années, le détroit de Gibraltar est aussi un point de rencontre entre le Nord et le Sud, au même titre que la frontière Mexique/Etats-Unis, canalisant, mais aussi accentuant, les peurs de l'Occident. Le drame des clandestins a pris une dimension médiatique nouvelle depuis les assauts migratoires autour de Ceuta et Melilla en 2005. Reconsidérée par les médias, l'immigration clandestine transméditerranéenne ne laisse plus indifférente. Pendant plus d'une décennie, le détroit a été la principale porte d'entrée de l'espace Schengen. Pour contrer le phénomène, les autorités espagnoles et européennes ont répondu en le verrouillant matériellement et en créant un cadre juridique nouveau. Le détroit de Gibraltar s'est imposé comme un véritable laboratoire, un modèle d'application des conventions de Schengen et de Dublin.

4 Par définition, un détroit fait communiquer deux espaces maritimes et des terres rapprochées. Le détroit de Gibraltar est avant tout un lieu de passage, on ne peut pas considérer son analyse en se limitant uniquement à ses deux rives. Le contrôle d'un détroit n'a aucune signification si on ne le perçoit pas comme un espace plus large et plus ouvert, un véritable carrefour. Appliquer une politique de fermeture à un tel espace n'a de sens seulement si on considère sa fonction polarisante au-delà de ses limites, en amont et en aval des flux qui le parcourent. Dans la géopolitique actuelle et de par sa position géographique, le détroit de Gibraltar s'est imposé comme le point nodal des flux migratoires euro-africains, mais aussi comme la centralité du partenariat euro-méditerranéen et de la politique de «frontièrisation» de Schengen. Depuis que l'Union européenne verrouille les frontières, sa politique migratoire se diffuse depuis le détroit sur l'ensemble du Maghreb, repoussant un peu plus encore les migrants en accentuant le mythe de la forteresse assiégée.

5 Assiste-t-on aujourd'hui au contournement du détroit par les filières clandestines ? Le limes érigé par l'Europe justifie l'ouverture de nouvelles voies de passage par défaut, le détroit conservant sa fonction polarisante dans le parcours des migrants. Une fois verrouillées par des moyens sophistiqués comme ceux mobilisés dans le détroit, ces 
nouvelles filières se rabattront probablement sur Gibraltar. Le détroit apparaît comme un espace modèle du phénomène migratoire aussi bien pour les moyens mis en œuvre par l'Europe pour contrer les flux que dans la réactivité des migrants à contourner la forteresse Schengen.

1. Enjeux et limites de l'approche statistique

6 L'analyse de l'ampleur des flux clandestins nécessite une approche chiffrée. Les statistiques pouvant rendre compte des migrations se basent sur le nombre de migrants et de pateras ${ }^{2}$ interceptés que publient annuellement le Ministère de l'Intérieur espagnol, le Secrétariat d'État à l'Immigration et l'Émigration et la Guardia Civil. Les seules données sur les interceptions ne traduisent pas pour autant le nombre de tentatives, le nombre de traversées réussies, ni même l'efficacité des dispositifs mis en place. Le recensement des disparus du détroit contribue à évaluer le phénomène. La Guardia Civil a comptabilisé entre 1997 et 2005, 1766 victimes contre plus de 3100 selon des ONG. L'Associación Pro Derechos Humanos de Andalucía recensait 1167 décès pour la seule année de 2006 dans le détroit et plus de 6000 au large des Canaries. Les régularisations massives de 2000-2001 et 2005 ont prouvé la perméabilité des frontières espagnoles. Une interprétation uniquement chiffrée des migrations illégales ne constitue donc pas une approche juste du phénomène.

7 Au regard de la figure 1, malgré les moyens techniques et juridiques mobilisés par l'Espagne depuis 1995, on remarque que le nombre de migrants arrêtés dans le détroit est au même niveau qu'il y a dix ans. L'évolution globale montre une croissance constante du nombre des migrants arrêtés. Doit-on en conclure que le verrouillage du détroit a su dissuader les migrants et qu'aujourd'hui ceux qui y transitent sont tous appréhendés par la Guardia Civil, ou, au contraire, constater l'échec des systèmes antimigratoires européens ? L'Espagne vante pourtant les mérites de son verrouillage : Alfredo Pérez Rubalcaba, ministre de l'Intérieur, affirme que «les flux d'immigration illégale dans le détroit sont désormais contrôlés, justifiant la pression en Afrique de l'Ouest ${ }^{3}$.

Figure 1 - Arrestations totales côtes espagnoles - Toutes nationalités

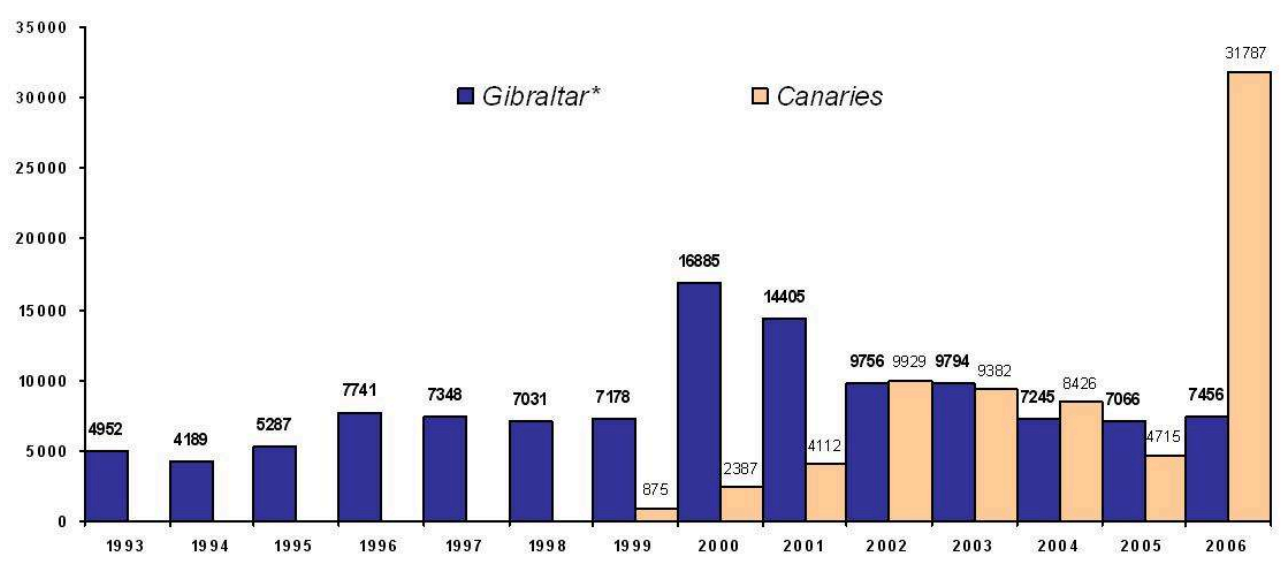

Source : Ministerio del Interior, Secretaría de Estado de Inmigración y Emigración et Guardia Civil ${ }^{4}$ 
Figure 2. - Pateras interceptées dans le détroit depuis 1996, selon le municipe
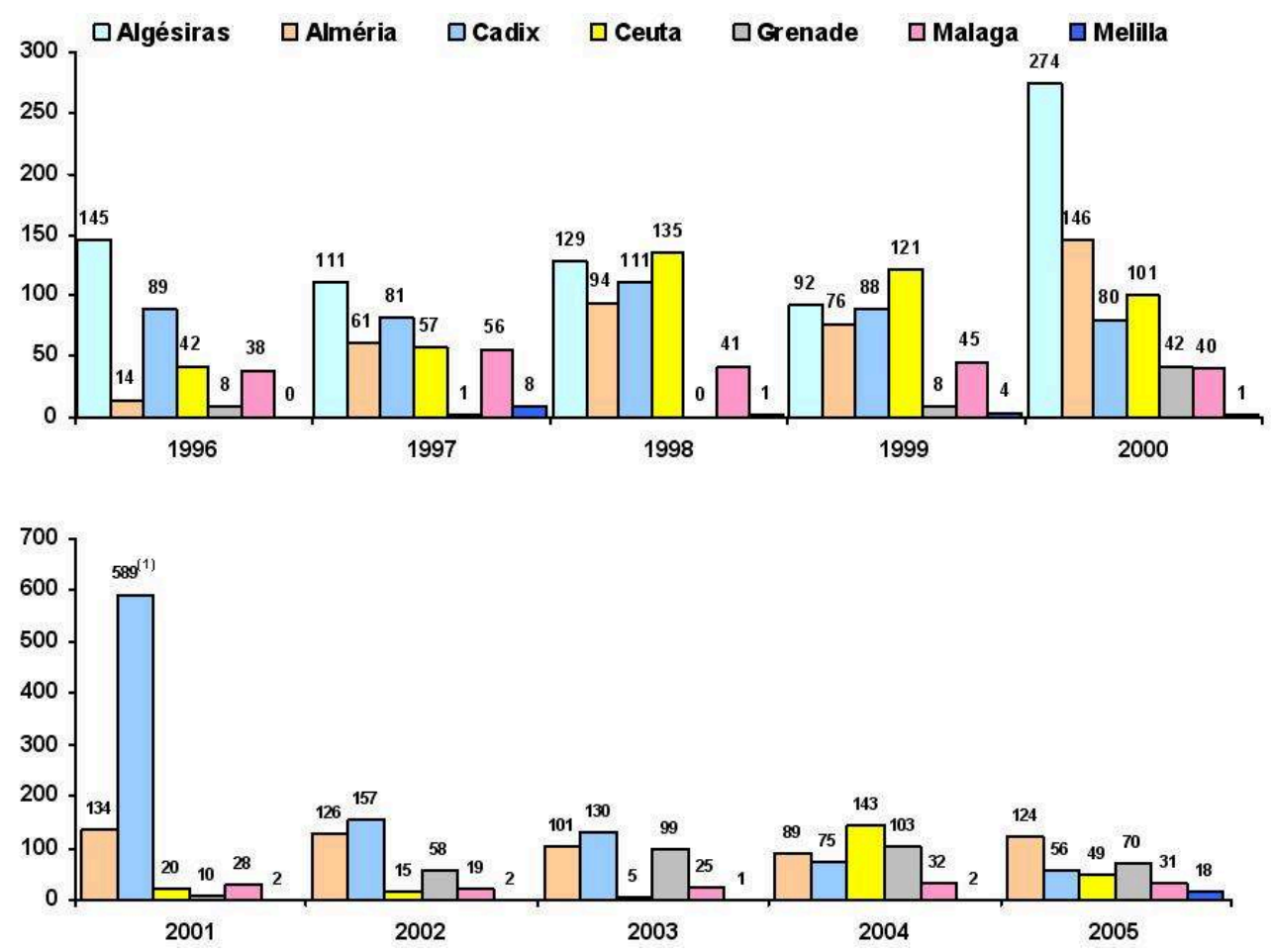

(1) Depuis 2001, les interceptions à Algésiras sont comptabilisées avec le municipe de Cadix Source: Guardia Civil

9 Parmi les autres données publiées, le nombre de pateras interceptées ne présente pas d'intérêt particulier si on ne considère pas le nombre de clandestins embarqués. En revanche, l'étude du lieu de leur interception montre la réactivité des migrants face à la surveillance du littoral en des points précis (figure 2). La province de Cadix et la région d'Algésiras qui captaient la quasi-totalité des flux, sont aujourd'hui contournées. Les provinces plus éloignées, considérées comme moins surveillées, polarisent désormais les migrations.

La figure 3 détaille les lieux d'arrestation des migrants. La Guardia Civil n'a plus publié ces données depuis 2000. Alors que la traversée en pateras représentait la méthode la plus utilisée par les clandestins, l'étude de données plus récentes met en exergue la greffe des parcours des migrants aux flux légaux qui traversent le détroit. En comparant ces données au nombre total de pateras interceptées dans le détroit (figure 2.1), on peut évaluer le nombre de passagers appréhendés par pateras. Ainsi, en 1997, en moyenne 6,8 passagers ${ }^{5}$ étaient arrêtés à bord d'une patera, contre plus de 21 en 2000. 
Figure 3 - Structure des arrestations des migrants clandestins selon le lieu d'interpellation

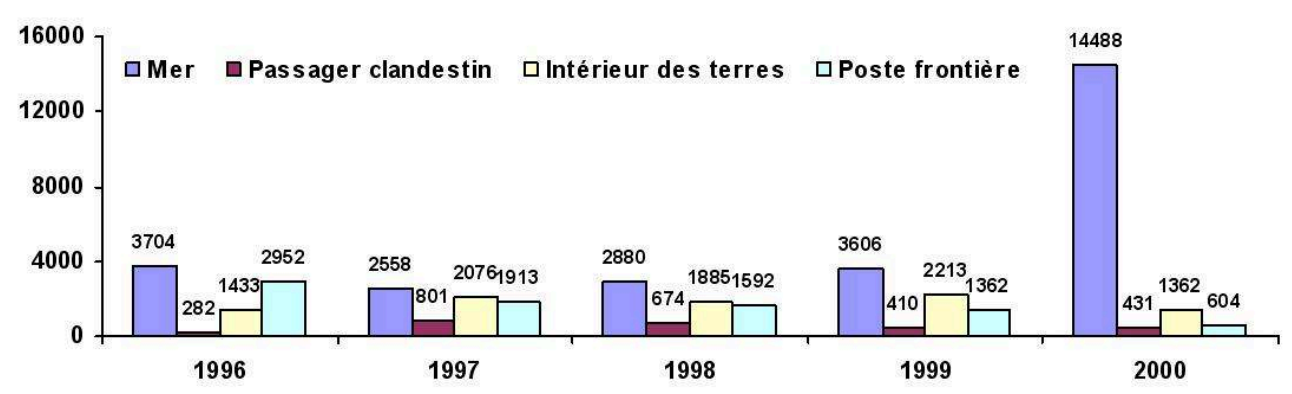

Source : Guardia Civil

Figure 4 - Structure des arrestations des migrants clandestins selon la nationalité

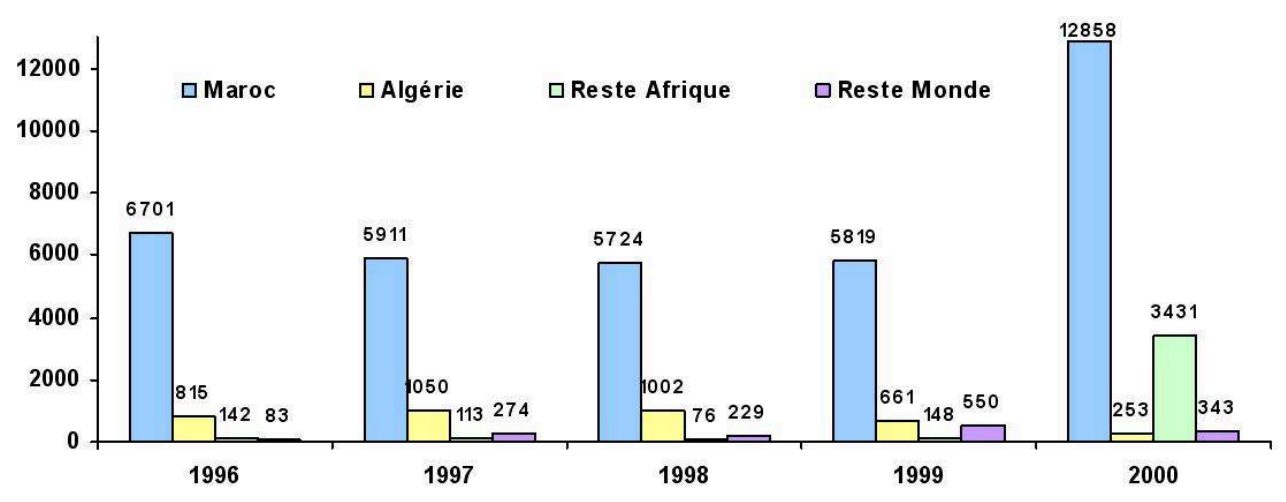

Source : Guardia Civil

11 La figure 4 montre l'évolution de la structure par nationalité. Ces données présentent un enjeu majeur dans la volonté européenne de conclure un accord de réadmission avec tel ou tel pays. Depuis 2003, l'effectif des migrants subsahariens interceptés dépasse celui des Marocains. Pourtant, l'Europe continue de considérer le Maroc comme principal responsable des migrations transméditerranéennes. La manipulation des chiffres et des images joue un rôle majeur dans la gestion des migrations irrégulières euro-africaines et implique des mutations juridiques, la mise en place d'une collaboration et la répartition des subventions européennes. Pour estimer les nationalités les plus représentées, il faut désormais s'appuyer sur les données du Ministère de l'Intérieur qui gère les expulsions et les rapatriements, les régularisations d'étrangers ou sur la nationalité des détenus des Centres d'Internement pour Étrangers.

\section{L'Espagne, dernier rempart de l'espace Schengen2.1. Le poids de Schengen}

Avec l'entrée de l'Espagne dans la CEE en 1986, le détroit de Gibraltar devient l'une des limites de l'Europe. Pendant les années 1980, le partenariat avec la rive sud de la Méditerranée constitue une priorité stratégique pour l'Europe, mais les difficultés économiques et l'ouverture à l'est, avec la chute du mur de Berlin, redéfinissent les rapports avec la Méditerranée. L'Europe entame un processus de "frontièrisation » de ses limites extérieures communes avec la signature de la convention de Schengen en juin 1985, ratifiée par l'Espagne en 1991 et mise en application en 1995. Schengen crée le mythe de la forteresse assiégée et transforme durablement la fonction de porte transversale du détroit de Gibraltar, érigeant un rideau de fer euro-africain. La convention de Dublin, entrée en vigueur le 1er septembre 1997, exige le traitement 
exclusif d'un individu en situation irrégulière par l'État qui a permis son entrée dans l'espace Schengen. Ce principe vise les pays méridionaux de l'Europe par où transitent les flux clandestins, en les invitant à rendre leurs frontières plus hermétiques ou, à défaut, à en assumer les conséquences.

En ratifiant ces accords, l'Espagne s'engage à contrôler son littoral et la frontière sud de l'Europe. Gibraltar devient le point nodal de la politique européenne de contrôle des frontières qui se met en place sur une double articulation entre outils juridiques et moyens techniques de surveillance ayant pour but de dissuader les migrants. En 1991, le visa devient obligatoire pour les ressortissants marocains souhaitant se rendre en Espagne. En 1989, la première patera s'échoue à Cadix. L'Espagne, pays d'immigration jusque dans les années 1980, opère une mutation juridique de sa politique migratoire. La première loi sur les étrangers intervient en 1985, la Ley Orgánica sobre Derechos y Libertades de los Extranjeros en España de juillet 1985, définissant pour la première fois leurs droits.

Face aux pressions européennes, la législation espagnole se durcit jusqu'à la signature d'accords de réadmission avec les pays d'origine des migrants. L'Espagne se dote de lois permettant l'expulsion des individus en situation irrégulière. Les modalités de rapatriement résultent de la loi organique avril 2000 du 11 janvier 2000 relative aux droits et aux libertés des étrangers ${ }^{6}$. Reconnue comme une "infraction administrative grave ", la présence illégale sur le territoire espagnol est sanctionnée d'une amende ou d'une expulsion. Une fois interpellé, un étranger en situation irrégulière est placé en rétention dans un centre d'internement pendant quarante jours. Si, pendant cette durée, la nationalité de l'individu n'est pas définie ou si son pays d'origine refuse de coopérer pour son rapatriement, il est remis en liberté avec un ordre d'expulsion inapplicable. En 2003, près de 40000 ordres d'expulsion n'ont pu être appliqués, 37000 en 2004 contre 13300 exécutés. En 2006, l'Espagne a rapatrié, refoulé ou expulsé 99445 étrangers en situation irrégulière ${ }^{7}$.

Sur le modèle de ses partenaires européens, Madrid fait construire des centres de détention pour clandestins. Parmi les dix Centres d'Internement pour Étrangers, deux se trouvent à Algésiras et Malaga ${ }^{8}$. Ceuta et Melilla sont dotées de Centres d'Accueil Temporaire pour Immigrés, l'équivalent des zones d'attente. En 2004, 24490 immigrants y étaient internés. Parmi les nationalités représentées, la part croissante des migrants originaires d'Asie caractérise l'attraction du détroit et le rôle de transit du Maroc à travers le monde.

2.2. Le SIVE ou le mur euro-africain

16 La seule création d'une juridiction répressive ne permet pas de contrer les flux de migrants. L'Espagne a donc investi à partir de 1990, plus de 65 millions d'euros dans la surveillance de son littoral. Madrid a multiplié les plans et les systèmes de surveillance. Malgré les investissements espagnols pour se rendre "eurocompatible ", l'immigration clandestine via le détroit de Gibraltar augmente à partir de 2000. L'Espagne annonce la création d'un contrôle électronique ultrasophistiqué permanent du détroit : le Système Intégré de Surveillance Extérieure (SIVE). Ce système s'appuie sur la coordination d'unités terrestres, maritimes et aériennes autour de technologies uniques (radars sensoriels, caméras thermiques et infrarouges) et surveille en continu les eaux du détroit.

17 Le SIVE entre en fonction en 2002 à Algésiras, avant d'être étendu à Tarifa et Malaga en 2003, Cadix et Huelva en 2004, Ceuta et Melilla en 2005 et Almeria en 2006. Le SIVE a au 
total coûté 260 millions d'euros et dispose aujourd'hui de 25 stations de surveillance. Il fonctionne en permanence et rend la traversée illégale du détroit théoriquement impossible. Sa fonction dissuasive a ainsi permis une baisse considérable des trafics de drogue et de contrebande. Le verrouillage du détroit constitue un modèle européen dans la lutte contre l'immigration clandestine. Les nouveaux points d'entrée de l'espace Schengen devraient se doter d'un tel système. Mais la simple logique de "frontièrisation » dépourvue d'une politique menée en amont des flux ou d'un appui au développement des pays émetteurs, permet seulement de repousser les trajectoires des clandestins vers des routes plus périlleuses ou les greffer aux autres flux qui parcourent le détroit.

2.3. Les paradoxes des régularisations massives

18 L'évolution démographique de l'Espagne laisse entrevoir un manque de main-d'œuvre dans de nombreux secteurs d'activité. La main d'œuvre étrangère constitue un élément moteur de l'économie du pays. En 2003, une enquête a révélé que le quart du PIB espagnol dépendrait des emplois occupés par des immigrants avec ou sans papier ${ }^{10}$. La volonté de mieux contrôler la production nationale et d'obtenir de nouvelles ressources fiscales et de nouvelles cotisations à la sécurité sociale, a conduit l'Espagne à réaliser cinq phases de régularisations en 1985-1986, 1991, 1996, 2000-2001 et 2005.

En 2005, l'Espagne a régularisé 573270 travailleurs en situation irrégulière. Alors qu'au niveau national, les secteurs d'activités les plus concernés ont été les employés de maison et du bâtiment, le secteur agricole a concerné plus de $52 \%$ des régularisés d'Andalousie ${ }^{11}$, marquée également par la grande proportion de ressortissants africains, renforçant la polarisation de l'activité maraîchère sous serre les flux euroafricains via Gibraltar. De telles régularisations massives entraînent des désaccords au sein de l'Union européenne. L'effet d'« appel d'air », provoqué par une telle politique, est généralement condamné par la droite européenne. L'annonce des régularisations de 2000 et 2001 avait entraîné une progression de $+135 \%$ des arrestations dans le détroit de Gibraltar (figure 1). Au $1^{\mathrm{er}}$ janvier 2007, selon l'Institituto Nacional de Estadística (INE), l'Espagne compte 3884573 étrangers, soit 8,4\% de sa population totale. En comparant les chiffres de l'INE ${ }^{12}$ à ceux du Ministère de l'Intérieur qui recensait 3021808 permis de résidence au 31 décembre 2006, on peut considérer qu'au moins 862765 individus se trouvent en situation irrégulière en Espagne. En Andalousie, l'INE comptabilise 488928 étrangers et le Ministère de l'Intérieur 362467 étrangers en situation régulière, soit au moins 126000 clandestins.

3. L'externalisation de la politique migratoire européenne3.1. Le chantage au développement

La « frontièrisation » de l'espace Schengen sur ses interfaces méridionales a répondu au souhait commun d'harmoniser les politiques migratoires et d'asiles des pays européens. Face à la difficulté de rendre ses frontières totalement imperméables, l'Union européenne s'attache à créer une zone tampon protégeant l'espace Schengen des migrants africains. La politique européenne de voisinage est aujourd'hui conditionnée par la gestion des flux migratoires. Les principes de la convention de Dublin de responsabilisation des pays de transit ont ainsi été appliqués aux pays du Maghreb.

21 Avec la complexification de l'accès direct à l'Europe, le Maghreb est devenu une zone de transit. En délocalisant ses frontières sur la faille méditerranéenne, l'Union européenne cherche à endiguer les flux migratoires jusqu'aux frontières du Maghreb, selon le principe des pays tiers sûrs. La logique de responsabilisation subie par 
l'Espagne au milieu des années 1990 a été reproduite aux pays maghrébins à travers une coopération dans le contrôle des frontières, l'instauration d'une législation " euroforme " et par la conclusion d'accords de réadmission. Pour obtenir les résultats escomptés, l'Europe s'est appuyée sur le Partenariat Euro-Méditerranéen et les Accords Euro-méditerranéens d'Association (AEA) ${ }^{13}$. L'objectif d'une coopération complète sur l'immigration illégale est devenu la thématique principale du partenariat, mais aussi la condition primaire de son bon fonctionnement. En obligeant les pays du Maghreb à accentuer la surveillance de leurs frontières en contrepartie d'aides financières, l'Union européenne instaure une politique de chantage d'aide au développement. Pour encourager le Maroc, l'Europe subventionne les grands travaux du nord du royaume, coordonnés par l'Agence pour la Promotion et le Développement des provinces du nord du Royaume ${ }^{14}$. Véritable priorité du pays dans sa volonté de s'intégrer aux réseaux d'échanges mondiaux, l'émergence du nord, interface vers l'Europe, passe par la restructuration économique du Rif, de Tanger (création du port Tanger-Méditerranée) et des villes de Tétouan ou Fnideq pour mettre fin à la polarisation autour de Ceuta. Ainsi, alors que l'Union européenne avait conditionné le fonctionnement du partenariat euro-méditerranéen autour d'une clause démocratique et du respect des droits de l'homme, la conditionnalité migratoire est devenue le compromis du programme de développement commun.

\subsection{Les conséquences d'une législation euroforme, l'émigration illégale}

La délocalisation des pratiques administratives, juridiques et policières prônées par la convention de Schengen a poussé à la responsabilisation des pays maghrébins par la création de lois et par la conclusion d'accords de réadmission. Jusqu'en 2003, la législation marocaine relative à l'entrée et au séjour des étrangers et à l'émigration et l'immigration irrégulière n'avait pas évolué depuis l'époque coloniale ${ }^{15}$. Face aux pressions européennes et à la situation interne liée aux attentats de Casablanca de mai 2003, le Maroc annonce l'adoption de deux lois : le 11 novembre 2003, la loi n 02-03 relative à l'entrée et au séjour des étrangers, à l'émigration et à l'immigration irrégulières, et le 28 mai 2003, la loi nº3-03 relative à la lutte contre le terrorisme. La présentation conjointe de ces lois conforte l'externalisation des craintes européennes et la volonté de créer aux marges de l'Europe, une zone tampon protégeant ses intérêts. Alors que les pays européens harmonisent leur législation, les pays maghrébins renforcent ou créent une approche répressive, parfois contraire à la Convention internationale sur la protection des droits des travailleurs migrants et de leur famille, pourtant signée par le Maroc en décembre 1990 à l'ONU.

La loi vise les migrants subsahariens qui transitent par le territoire marocain, mais aussi les personnes tentant de quitter illégalement le Maroc, constituant une approche législative inédite marquée de l'influence européenne. Calquée sur les lois européennes, principalement françaises, la loi de février 2003 prévoit la nécessité pour chaque étranger de posséder des papiers en règles et de justifier les motifs de sa venue. Deux titres de séjour sont créés: la Carte d'Immatriculation, octroyée aux travailleurs étrangers, et la Carte de Résidence, délivrée aux étrangers ayant justifié une période ininterrompue de résidence de quatre ans, aux conjoints ou parents d'un ressortissant marocain ou aux réfugiés. Si les motifs de séjour sont jugés insuffisants ou si l'individu représente "une menace pour l'ordre public », l'entrée du territoire lui sera refusée. Dans le cas d'une expulsion inapplicable (femme enceinte, mineur, résident permanent depuis six ans ou réfugié politique), l'étranger est placé dans un centre de rétention 
administratif. Des amendes et jusqu'à six mois d'emprisonnement sont prévus pour toute personne ayant pénétrée ou tentée de pénétrer le territoire illégalement.

Des sanctions sont également prévues pour les individus qui quittent illégalement le pays, qu'ils soient Marocains ou non. L'article 50 punit toute personne qui tente d'émigrer clandestinement d'une amende de 3000 à $10000 \mathrm{DH}$ et d'une peine de un à six mois d'emprisonnement. La corruption est également visée par ce projet : une amende de 50000 à $500000 \mathrm{DH}$ et de deux à cinq ans de prison (article 52) sont prévus pour les représentants de la loi convaincus d'avoir assisté des passeurs, et de six mois à trois ans de prison et d'une amende de 50000 à $500000 \mathrm{DH}$ pour toute personne ayant organisé un départ clandestin. Les passeurs risquent même jusqu'à quinze ans de prison si un de leur «client » venait à être blessé et jusqu'à la perpétuité s'il venait à décéder.

L'application de la loi de février 2003 au Maroc conditionne le respect des Droits de l'Homme et des Migrants. Les centres militaires de rétention pour étrangers ne sont pas accessibles aux ONG. Les expulsions vers la frontière algérienne, vers la ville d'Oujda, se font en masse, contrairement à l'article 22 de la Convention internationale qui interdit les expulsions collectives. Les manquements de la législation marocaine affirment le rôle du pays comme sentinelle au service de Schengen, renforçant le sentiment de frustration omniprésent dans la société : "on nous refuse l'existence et, dans le même temps, on nous interdit de nous en aller"; "la marine royale ne protège plus les côtes marocaines contre l'étranger, elle protège l'étranger contre les Marocains " ${ }^{16}$.

3.3. La sous-traitance migratoire et les accords de réadmission

L'AEA Maroc/Union européenne de 1996 prévoyait à terme la signature d'un accord de réadmission global pour tous les migrants illégaux, marocains ou non, se trouvant dans l'espace Schengen et ayant transité par le Maroc. Rabat a d'abord refusé d'endosser la responsabilité des flux, mettant en exergue la part des Subsahariens qui transitent par son territoire. L'Union européenne s'est résolue à financer l'amélioration de la surveillance du littoral marocain, mais a contraint le pays à la signature d'accords de réadmission bilatéraux. En 1992, l'Espagne et le Maroc signent un premier accord qui s'applique seulement aux Marocains. Le renouvellement de l'accord en 1996 permet l'application de la clause stipulant que tous les migrants entrés illégalement en Espagne via le Maroc y sont reconduits. Selon la loi espagnole, l'expulsion ne prend effet que si l'identité du migrant est établie sous quarante jours et l'expulsion des mineurs est également régie par des clauses spécifiques.

L'accord hispano-marocain ne répond pas à la volonté de l'Europe de conclure un accord global. Rabat souhaiterait que les pays de l'Union européenne signent directement des accords avec les pays d'Afrique subsaharienne, refusant d'endosser le rôle de gendarme et de débarras migratoire, et d'assumer la responsabilité des migrants qui transitent par son territoire et qui y séjournent de plus en plus. Pour la première fois en 2003, le nombre de migrants subsahariens arrêtés alors qu'ils tentaient de passer du Maroc en Espagne a dépassé celui des Marocains. La stratégie du Maroc de se poser en victime de ces flux pousse l'Espagne à la conclusion d'accords de réadmission avec les pays sub-sahariens.

L'absence d'accord harmonisé entre l'Union européenne et les pays émetteurs contribue à multiplier les spécificités qui favorisent les contournements juridiques. Le Maroc refuse la négociation d'un accord global et s'affaire à la conclusion d'accords avec les pays subsahariens. Le Maroc marchande désormais sa politique d'endiguement 
migratoire. En instrumentalisant le désespoir des migrants par la diffusion d'images spectaculaires des assauts contre les remparts des présides, Rabat a fait pression sur ses partenaires européens pour recevoir des aides supplémentaires.

Les accords de réadmission et le chantage au développement instauré par l'Europe ont profondément modifié les relations entre le Maroc et ses partenaires européens, dépassant, avec cynisme, le drame humain qui se joue aux frontières de l'espace Schengen. La gestion des flux migratoires est devenue la source d'un chantage diplomatique permanent. La conclusion d'un accord de pêche, la concurrence maraîchère, les trafics de drogue et de contrebande, les revendications territoriales (présides, îlot du Persil) ou l'avenir diplomatique du Sahara Occidental sont autant de sujets de désaccords dont le règlement est aujourd'hui conditionné par la gestion des flux migratoires qui transitent par le Maroc.

4. Des filières migratoires réactives ou l'inefficacité du verouillage européen?

L'afflux record enregistré aux Canaries en 2006 et la déferlante migratoire autour de Ceuta et Melilla en 2005 ont sensibilisé les médias européens à la question migratoire. Certes, depuis la mise en place du SIVE, le nombre de migrants qui transitent par Gibraltar a diminué, mais de nombreux clandestins continuent de tenter sa traversée en contournant le dispositif Schengen. Quelles réalités masquent, ou dévoilent, les statistiques annuelles des arrestations de la Guardia Civil? Combien de migrants atteignent réellement l'Espagne?

31 La phase de régularisation de 2005 a montré les limites du caractère dissuasif des lois espagnoles et de la surveillance des frontières. L'arsenal juridique répressif espagnol, européen et marocain, les moyens techniques du SIVE ou la collaboration des polices auraient du repousser les migrants. Au contraire, ils contournent désormais ces systèmes en utilisant les failles juridiques des accords de réadmission, en ouvrant d'autres routes migratoires, en se greffant aux flux légaux qui parcourent le détroit, en rabattant leur désir d'Europe sur Ceuta et Melilla ou en profitant des politiques de régularisation massive.

4.1. Contourner le SIVE, l'Europe en camion

32 Les modestes pateras ont été remplacées par des embarcations motorisées qui traversent le détroit bien plus chargées, expliquant en partie la constante diminution du nombre de barques interceptées et la relative stabilité du nombre de migrants arrêtés dans le détroit. Le SIVE a également rebuté les clandestins, mais le détroit de Gibraltar a conservé sa fonction polarisante. Les conteneurs et les camions sont devenus la voie de transit privilégiée des migrants marocains qui se faufilent dans les bateaux qui partent pour l'Europe.

Chaque année, 40000 camions transitent depuis Tanger vers Algésiras, 15000 vers le port de Cadix et à peine $35 \%$ peuvent être fouillés. En 2002, les autorités du port de Tanger-Méditerranée avaient fait part de l'arrestation de 11200 migrants prêts à embarquer vers l'Europe. Les médias marocains relèvent régulièrement l'arrestation de migrants asiatiques et subsahariens, renforçant la polarisation de la péninsule tingitane dans les flux clandestins. Face au risque de perdre l'influence du port de Tanger, le Maroc a obtenu des fonds européens pour améliorer les contrôles (scanners et détecteurs thermiques) et la construction d'une protection de six mètres de haut autour du port.

34 En 2002, dans le port d'Algésiras, 985 clandestins ont été appréhendés, 1517 en 2004 et 3687 en 2005. Cette augmentation $(+191 \%)$ caractérise la perméabilité du port de 
Tanger, mais aussi la faible dimension dissuasive de la surveillance européenne. Les flux des migrants se calquent désormais sur les réseaux d'échanges légaux. Alors qu'avant 2000, à peine $5 \%$ des arrestations de migrants se faisaient à bord des camions et porte-conteneurs, l'analyse du nombre d'arrestations dans le port d'Algésiras et des arrestations globales sous-entend que près de $50 \%$ des migrants interceptés en 2005 auraient transité par les ports du détroit.

Le retour estival des ressortissants marocains de l'étranger (RME), lors de l'Opération Transit, engendre en quelques jours $55 \%$ du trafic annuel des passagers et $70 \%$ de celui des voitures. En 2006, près de 2,7 millions de personnes et 700000 véhicules ont transité par le détroit. Le retour en Europe, à la fin du mois d'août, constitue une période critique pour les douaniers espagnols. Le kif et les clandestins se mêlent aux RME. Ainsi, en 2003, à la fin du mois d'août, 270 clandestins ont été arrêtés dans le port d'Algésiras et 875 en 2005.

4.2. Les mineurs isolés

36 Alors que le premier accord de réadmission hispano-marocain ne permettait pas l'expulsion des mineurs isolés, les deux pays s'entendent, lors de son renouvellement en 2003, pour le rapatriement des mineurs non accompagnés si leur famille a été préalablement identifiée. Depuis 1996, la Guardia Civil a relevé une constante augmentation du nombre de mineurs arrivés clandestinement et isolés en Espagne à bord d'une patera ou d'un camion. En 1998, 382 étaient hébergés dans des centres d'accueil, 705 en 1999 et 1143 dès 2000. Dans les provinces espagnoles du détroit, 1071 entrées illégales de mineurs ont été enregistrées en 2004, 2365 en 2005. L'Espagne négocie depuis avec le Maroc pour mettre fin à ce vide juridique. En avril 2006, l'Espagne propose de cofinancer des centres de réinsertion pour les mineurs marocains «non expulsables». À défaut d'avoir pu identifier leur famille, les enfants sont remis aux autorités. L'accord prévoit la construction de deux centres, dont un se situera à Tanger d'où proviendraient près de $67 \%$ des mineurs clandestins isolés ${ }^{17}$. Plus de 5000 mineurs recensés dans les centres d'accueil sont, ou devraient être, concernés par ces expulsions. En 2006, 111 mineurs, dont $80 \%$ étaient d'origine marocaine ${ }^{18}$, ont ainsi été rapatriés. Les expulsions sont ralenties par le manque d'infrastructures et par le respect des Droits de l'Enfant dont l'Espagne et le Maroc sont signataires. De plus, la loi marocaine de février 2003 (article 26) ne sanctionne pas l'émigration des mineurs.

4.3. Les nouvelles routes de l'immigration clandestine, parcours migratoire par défaut

Le verrouillage complet du détroit par le SIVE pousse les candidats à l'exil à ouvrir de nouvelles routes migratoires plus longues et plus dangereuses. Ainsi, la mise en place du SIVE avait détourné les flux migratoires vers les municipes de Grenade et d'Almeria. Depuis 2002, près de $55 \%$ des pateras qui traversent le détroit sont interceptées dans ces provinces contre 6,6\% en 1996 (figure 2). Plus éloignées du détroit, de nouvelles voies migratoires par défaut ont émergé. Ainsi, en 2000, les Canaries ont enregistré 2300 arrivées illégales, 8500 en 2004 et plus de 31000 en 2006. Sur les îles italiennes au large de la Tunisie 13000 arrivées ont été comptabilisées en 2004, 23000 en 2005 et près de 26000 en 2006, principalement depuis la Libye. La focalisation des efforts européens sur l'Andalousie explique le détournement des flux clandestins vers ces nouvelles routes.

38 Les moyens techniques et juridiques mobilisés par Schengen n'ont pas su dissuader les candidats à l'exil. L'Europe peut donc continuer à ériger des barrières sans que le flot de clandestins ne se tarisse. La fermeture complète de l'espace Schengen contribuera à 
diversifier les voies d'entrée en Europe, mais le détroit de Gibraltar restera le point nodal des flux. Le projet d'une liaison fixe à travers le détroit risque de reproduire le schéma du camp de Sangatte aux abords de l'Eurotunnel. Avant que l'Europe n'érige la forteresse Schengen, les courants marins et les vents violents du détroit n'avaient pas rebuté les pateras, la volonté européenne d'endiguer le phénomène risque donc de rester impuissante.

5. Ceuta, forteresse assiégée

39 La polarisation des flux migratoires subsahariens vers la péninsule tingitane relève également de l'attraction des deux villes espagnoles ancrées sur le continent africain. Les présides de Ceuta et Melilla ${ }^{19}$ constituent des avant-gardes de la forteresse Schengen et renforcent l'attrait des rives du détroit pour les migrants africains.

Les accords de réadmission de l'Espagne avec l'Algérie et le Maroc rendent quasiimpossible l'entrée des Maghrébins dans les enclaves. Pourtant, un accord frontalier permet aux Marocains de la province de Tétouan de se rendre à Ceuta pour travailler ou consommer, créant un flux quotidien de plus de 20000 personnes au poste frontière du Tarajal, unique point d'entrée de la ville. Cet intense trafic facilite l'entrée de clandestins qui tentent ainsi de s'infiltrer dans le port pour rejoindre l'Europe. Comment l'Europe peut-elle tolérer une telle spécificité frontalière, alors qu'elle prône l'imperméabilité de ses frontières?

41 Jusqu'aux premiers accords de réadmission entre l'Espagne et les pays d'Afrique subsaharienne et l'application de l'accord hispano-marocain stipulant l'expulsion vers le Maroc de tous les clandestins ayant transité par celui-ci, l'enclave de Ceuta fut très prisée par les migrants subsahariens. En 2005, le renforcement de la collaboration euromarocaine et la multiplication des arrestations de Subsahariens poussent les migrants subsahariens à lancer des assauts massifs contre les grillages des présides. Dans la nuit du 29 septembre, cinq migrants sont tués aux abords de Ceuta et le 06 octobre, six sont abattus devant Melilla. L'Espagne lance sa "guerre aux migrants » et déploie l'armée dans les deux villes. La médiatisation de ces assauts donne une visibilité inédite aux migrations euro-africaines, articulant le désespoir de l'Afrique et les peurs européennes. Comme Mouammar Kadhafi délaisse de temps à autre la surveillance du littoral libyen, le Maroc instrumentalise ces assauts pour obtenir des aides supplémentaires de l'Europe.

5.1. Ceuta, laboratoire de la frontièrisation européenne

42 Comme pour le détroit de Gibraltar et les îles Canaries, l'Europe a rendu l'Espagne responsable du verrouillage de ses enclaves. Un seul poste frontière relit désormais l'enclave au Maroc ${ }^{20}$. Pour un coût de 30 millions d'euros, Ceuta est depuis 1995 entourée d'un "périmètre de sécurité ", financé à 75 \% par l'Union européenne. Long de huit kilomètres, le mur de protection, la verja ${ }^{21}$, est constitué d'une double barrière de barbelés. Régulièrement rehausséz ${ }^{2}$, le double grillage atteint aujourd'hui six mètres de haut. La zone séparant les grillages est militarisée depuis 1997. Le mur avance également en mer pour protéger l'enclave des pateras qui entrent dans les eaux du préside. Enfin, les autorités ont annoncé fin 2006, la création d'un mur de protection de 1300 mètres et de quatre mètres de hauteur autour du port.

43 Face à l'afflux de clandestins, un centre de rétention a été ouvert en 1997. Le centre de Calamocarro, "purgatoire» avant l'Europe, permet l'application des lois et des procédures d'expulsion. Prévu pour 700 personnes, il a parfois abrité jusqu'à 3000 personnes pour des structures inadaptées. Comme le Maroc se pose aujourd'hui en 
victime des migrations, l'Espagne a parfois surestimé l'occupation du camp pour obtenir des aides supplémentaires. Semblable à un camp de réfugiés, Calamocarro est aujourd'hui une priorité des clandestins qui ont pénétré l'enclave pour éviter une reconduite immédiate à la frontière.

La situation des enclaves symbolise l'évolution de la gestion des flux migratoires d'une Europe marquée par sa "frontièrisation ", par des politiques d'expulsion et leurs répercussions sur les lois marocaines. Véritable « laboratoire» ${ }^{23}$ de l'externalisation de la politique migratoire européenne, Ceuta est aussi le symbole de son inefficacité et du refoulement de la misère africaine aux marges de l'Europe et aux confins du Sahara. En 2005, 20000 migrants ont tenté de franchir les barbelés qui entourent les deux villes espagnoles ; officiellement, 2324 avaient réussi à pénétrer dans Ceuta. La majorité des migrants, après avoir subi les quarante jours de détention légaux et reçu un avis d'expulsion inapplicable, ont pu se rendre en Espagne. En 2006, 1351 migrants ont réussi à entrer illégalement à Ceuta via le poste frontière, et seulement une tentative de franchissement a été enregistrée par la Guardia Civil.

\subsection{Ben Youlnech et Mesnana, aux marges de l'Europe}

La pression migratoire structure la frontière de Ceuta, mais elle conditionne aussi la vie des migrants subsahariens qui espèrent pénétrer dans l'enclave. La loi marocaine 02-03 a profondément modifié la présence étrangère dans le pays. À Tanger ou Tétouan, les immigrés vivent en marge de la société et subissent d'importantes discriminations. La plupart se sont regroupés dans des campements informels aux abords de Ceuta dans les forêts de Ben Youlnech et de Mesnana. Sujets à des rafles successives, la police marocaine a fait évacuer et expulser la quasi-totalité des occupants après les évènements de 2005.

Les expulsions de plus en plus fréquentes des étrangers en situation illégale du Maroc et la «frontièrisation " de l'Europe ont indirectement introduit la problématique de l'intégration des migrants subsahariens. L'étape marocaine se prolonge pour de nombreux migrants et devient une finalité pour d'autres. Le carrefour migratoire marocain a donc subi l'introduction d'un cosmopolitisme par son sud. La confrontation avec l'altérité aussi bien pour les trans-migrants subsahariens que pour la société marocaine crée des rapports conflictuels inédits. Les portes de Ceuta et Melilla se sont refermées et de nombreux Subsahariens doivent aujourd'hui repenser leur projet migratoire. L' "infranchissabilité » théorique des murs de Ceuta explique la légère augmentation du nombre d'arrestations dans le détroit de Gibraltar depuis 2005. Mais la majorité des clandestins subsahariens reporte leur projet migratoire vers les villes du sud, avec pour nouvel objectif les îles Canaries.

Conclusion

47 En 1990, lors d'un sommet hispano-marocain Félipe Gonzalez, alors premier ministre espagnol, déclarait « les contrôles aux frontières ne suffisent pas. Si j'avais entre 18 et 25 ans, et si je vivais de l'autre côté du détroit avec un revenu dix fois moindre que le SMIC européen, pour moi, il n'y aurait pas de frontières. J'arriverais à franchir toutes les frontières, tous les murs ». Depuis, l'écart de niveau de vie n'a cessé de croître et la volonté inébranlable d'émigrer en Europe a démontré l'impuissance du verrouillage des frontières. En dix ans, le détroit de Gibraltar est devenu la concrétisation et le modèle du processus de «frontièrisation » européen, mais aussi le symbole de ses limites et de la réactivité des migrants. Gibraltar, convoitise des migrants, est-il pour autant la porte de l'eldorado 
européen? L'espoir des milliers de migrants en attente au Maroc se situe-il sous les serres d'Andalousie?

Par ses interconnexions, Gibraltar condense les tensions qui parcourent le monde méditerranéen. Les relations euro-maghrébines sont aujourd'hui régies par la gestion des flux migratoires. La convention de Schengen a restructuré le limes africain. Le détroit de Gibraltar a capté l'influence juridique de l'Europe communautaire. Ses rives ont subi une réorganisation qui lui a donné une signification nouvelle. Son évolution récente à ramener ce point nodal du globe à son éternelle problématique, partagé entre verrou, synapse et frontière. Depuis ses rives, l'Europe diffuse sa volonté de se verrouiller. Tel un espace modèle, il démontre les capacités de l'Europe à surveiller son littoral et la frontière de ses dernières colonies africaines. Alors que des milliers de migrants ont disparu dans les eaux du détroit, le mur de Ceuta, pourtant modèle précurseur de la future frontière Mexique/Etats-Unis, symbolise toute l'impuissance du Nord à endiguer les flux migratoires.

De synapse euro-africaine, le détroit de Gibraltar en est devenu le verrou théorique. Mais fermée, interdite ou verrouillée par des moyens de plus en plus sophistiqués, une frontière reste perméable. Même si les autorités espagnoles vantent le verrouillage du détroit, son rôle polarisant n'a pas été affecté au regard de l'évolution récente du nombre d'interpellations. Certes le SIVE, les politiques d'expulsion et de réadmission permettent de mieux gérer les flux, mais ils ne détournent pas pour autant la pression migratoire. L'état de verrou du détroit a pour double conséquence l'ouverture par défaut de nouvelles routes migratoires et le contournement des spécificités législatives européennes, mettant ainsi en exergue la réactivité des migrants. En doublant ses efforts depuis le Maghreb, l'Europe se déresponsabilise du sort des migrants et transforme le Maroc en une impasse migratoire, redéfinissant son influence géopolitique sur l'ensemble de l'Afrique.

En impliquant les pays maghrébins dans leur lutte contre les migrations illégales, les pays de l'Union européenne les ont poussé à se positionner en victime. Rabat use aujourd'hui de ce statut pour influencer ses relations avec l'Europe, imposant à son tour un chantage migratoire. Le Maroc et la Libye ont accepté de devenir les sentinelles de l'Europe mais conservent leur capacité à réguler les flux, voire à les manipuler.

51 Le Maroc reporte aujourd'hui les principes de Schengen au-delà du Sahara. Les flux migratoires transsahariens et transméditerranéens et leur gestion ont redéfini la géopolitique du Maghreb. L'influence européenne tend à isoler le Maghreb du reste du continent africain, en le maintenant dans une sphère d'influence méditerranéenne. En acceptant de reproduire le schéma de fermeture appliqué dans le détroit le long de leurs frontières sahariennes, les états maghrébins réalisent le projet européen de constituer une zone tampon aux marges de l'espace Schengen. L'Europe constitue par le biais de sa politique migratoire une hiérarchisation transversale de sa périphérie africaine. En reconnectant l'Afrique arabo-berbère et l'Afrique subsaharienne à travers les flux migratoires transsahariens à vocation transméditerranéens, l'Union européenne pose indirectement la problématique de l'africanité du Maghreb.

La gestion des migrations transméditerranéennes implique des restructurations géopolitiques à plusieurs échelles qui se diffusent depuis le détroit de Gibraltar. De nouvelles régions émettrices de flux et de nouvelles destinations sont apparues en marge du détroit. Mais, par sa capacité à capter et à rediffuser, le détroit de Gibraltar impose encore sa centralité dans les migrations euro-africaines. Le contournement 
systématique par les migrants des systèmes mis en œuvre par l'Europe maintient le détroit parmi les points nodaux de ces flux. Même si la politique européenne de fermeture est alimentée par la pression des migrants, la reproduction permanente d'un schéma répressif et l'ignorance des inégalités, qui s'amplifient de part et d'autre du détroit, continueront de conforter la logique de politique anti-migratoire au détriment d'un développement des sociétés africaines.

\section{BIBLIOGRAPHIE}

\section{Bibliographie}

Baba N., 2006. Mineurs marocains non accompagnés : quelle réalité pour le retour?, Casablanca, Institut Universitaire Européen et Université Hassan II.

Bensaad A., 2005 Les migrations transsahariennes, une mondialisation par la marge, Maghreb-

Machrek, $n^{\circ} 185$, p. 13-36.

Clochard O., 2003, La Méditerranée, dernière frontière avant l'Europe, Les Cahiers d'Outre-Mer, $n^{\circ} 222$.

Elmadmad K., 2004. La nouvelle loi marocaine du 11 novembre 2003 relative à l'entrée et au séjour des étrangers au Maroc, et à l'émigration et l'immigration irrégulière, Casablanca, Institut Universitaire Européen et Université Hassan II.

El Yazami D., 2002. La forteresse assiégée et les nouvelles réalités migratoires in Le goût amer de nos fruits et légumes - L'exploitation des migrants dans l'agriculture intensive en Europe, Limans (Suisse), publication du FCE.

Jay S., 2001. Tu ne traverseras pas le détroit, Paris, n³29, Mille et une nuits.

Perrin D., 2005. Le Maghreb sous influence : le nouveau cadre juridique des migrations transsahariennes, Maghreb-Machrek, $\mathrm{n}^{\circ}$ 185, p. 59-80.

Wender A.S., 2004. La situation alarmante des migrants subsahariens en transit au Maroc et les conséquences des politiques de l'Union Européenne, Paris, CIMADE.

Migreurop, 2006. Le livre noir de Ceuta et Melilla - Guerre aux migrants, Paris.

Comisaria general de extranjeria y documentation, 2003. Rapport sur les Centros de Internamiento de Extranjeros, Madrid.

Ministerio de Trabajo y de asuntos sociales - Secretaria de estado de inmigracion y emigracion, 2003, 2004, 2005, Datos de inmigración irregular por medio de embarcaciones, Madrid.

\section{NOTES}

1. Les Almohades, dynastie régnante d'Al-Andalus entre 1147 et 1269, apparentaient le détroit à un canal.

2. Le terme patera désigne les embarcations à fond plat traditionnellement utilisées par les pêcheurs. Les premiers clandestins qui traversèrent le détroit ont utilisé ces 
embarcations ; le terme est depuis utilisé pour toutes les embarcations clandestines. La presse espagnole utilise également cayuco qui signifie pirogue.

3. Source : El País, 21 février 2007, Madrid

4. Les statistiques de Gibraltar regroupent les arrestations dans les eaux territoriales espagnoles, et celles effectuées dans les provinces de Cadix, Malaga, Grenade, Alméria, ainsi que les enclaves de Ceuta et Melilla.

5. Pour obtenir ce chiffre, on rapporte le nombre de clandestins arrêtés en mer en 1996 (figure 3) au total des pateras interceptées dans les eaux du détroit la même année (figure 2.1), soit 375 en 1996.

6. Ce texte a depuis été modifié. La loi organique $8 / 2000$ de décembre 2000 introduit la possibilité d'expulser les étrangers en situation irrégulière, alors qu'ils étaient seulement passibles d'une amende administrative (4/2000). Les modalités d'expulsion sont spécifiées dans le décret $n^{\circ} 2393$ du 30 décembre 2004. D'autres modifications ont été fixées avec la loi organique 11/2003 du 29 septembre 2003 et 14/2003 du 20 novembre 2003.

7. Selon le Bilan Migratoire présenté par Alfredo Pérez Rubalcaba, ministre de l'Intérieur. En 2006, 14068 immigrants ont été rapatriés dans leur pays ; 19273 immigrants ont été refoulés aux postes frontières $(+26,3 \%$ par rapport à 2005) ; 21216 immigrants ont été reconduits à la frontière $(+47,66 \%) ; 11567$ ont été expulsés pour des infractions à la ley Extranjería $(-5,14 \%)$ et 47389 migrants ont été reconduits en France.

8. En 2003, après avoir établi leur nationalité, 580 immigrés ont été expulsés depuis Algésiras ; 2232 expulsions ont été enregistrées à Malaga. À Algésiras, $96 \%$ des étrangers détenus provenaient d'Afrique subsaharienne. (Source : Comisaría General de Extranjería y documentación - Estadistica 2003)

9. Expression utilisée par José Maria Aznar.

10. Enquête tirée de Papeles de la Economía Española, deuxième trimestre 2003.

11. Source : Ministerio de Trabajo y Asuntos Sociales, Anuario 2005.

12. Pour comptabiliser les étrangers, trois méthodes sont utilisées et combinées : l'INE se base sur les inscriptions municipales (el padrón). Les chiffres du Ministère de l'Intérieur reposent sur les permis légaux de résidence et le Ministère du Travail s'appuie sur les chiffres de l'affiliation à la sécurité sociale. La différence des données de l'INE et du Ministère de l'Intérieur représenterait les clandestins.

13. La Déclaration de Barcelone de 1995 instaure le partenariat euro-méditerranéen entre les pays du pourtour méditerranéen et l'Union Européenne autour d'un un volet économique et financier, un programme politique et de sécurité et un volet social, culturel et humain. Le fonctionnement du partenariat est régi par une clause de conditionnalité démocratique. Le partenariat a pour but de créer à terme un espace commun de paix et de stabilité et une Zone de Libre Échange Euro-Méditerranéenne (ZLEEM). Cette construction se réalise en étapes à travers des AEA entre l'Union Européenne et chacun des autres pays membres. Après la Tunisie, le Maroc signe un AEA en 1996 qui entre en application le $1^{\mathrm{er}}$ mars 2000.

14. L'APDN a été crée en 1995 par Hassan II pour coordonner les grands projets structurels du nord du Maroc.

15. Trois textes de lois régissaient alors la vie des étrangers : le dahir (décret royal marocain) du 15 novembre 1934, du 02 janvier 1940, du 16 mai 1941, du 17 septembre 1947 et celui du 08 novembre 1949.

16. Jay Salim, 2001 
17. Baba Naïma, 2006

18. Données publiées par le Ministère de l'Intérieur.

19. Ceuta et Melilla sont deux enclaves espagnoles situées au Maroc. Ceuta appartient à l'Espagne depuis 1580 (les Portugais l'occupaient depuis 1415) ; Melilla est occupée depuis 1497. Avec les autres presides menores, les deux villes font partie intégrante de l'Union Européenne et de l'espace Schengen au même titre que l'Espagne.

20. Le poste frontière de Benzu, au nord de l'enclave, a été fermé en 2003.

21. Le terme verja signifie grille.

22. En 1995, le projet prévoyait un mur de barbelé de 2,5 mètres de haut. Il a été élevé à 3.5 en 1999. Il fait aujourd'hui 6 mètres de haut, mais devrait être rehaussé à 9 mètres sur les points les plus sensibles. Sa hauteur n'est pas régulière sur les $8,3 \mathrm{~km}$ de frontières. Trente tours servant de postes de contrôles et plusieurs caméras thermiques renforcent le dispositif.

23. Migreurop, 2006

\section{RÉSUMÉS}

Les assauts sur les grillages de Ceuta et Melilla à l'automne 2005 et le flux continu de migrants vers les îles Canaries en 2006 (+574\% par rapport à 2005) ont, par leur surmédiatisation, défini une nouvelle perception du phénomène migratoire. Depuis le début des années 1990, le détroit de Gibraltar, marge naturelle de l'Europe, s'était affirmé comme le point nodal, la centralité, de l'immigration clandestine transméditerranéenne. Il capte et diffuse, depuis l'apparition de la frontière commune de l'Europe Schengen, toutes les évolutions juridiques et techniques mises en place par l'Union européenne pour lutter contre les migrations illégales. Véritable laboratoire de l'externalisation de la politique migratoire européenne et de la coopération maghrébine, le détroit de Gibraltar s'est imposé comme l'espace modèle de la "frontièrisation » de l'espace Schengen, mais aussi le symbole de son inefficacité. La réactivité des migrants et leur capacité à contourner les dispositifs européens soulignent l'impuissance d'une politique de fermeture menée sans projet de développement des pays émetteurs.

As they received much media coverage, the assaults on the fences at Ceuta and Melilla in autumn 2005 and the continuous flow of migrants to the Canary Islands in 2006 defined a new perception of migratory phenomena. Since the beginning of the 90's, the Strait of Gibraltar, the natural limit of Europe, had become the nodal point, the very centre, of transmediterranean illegal immigration. Since the apparition of the common frontier of Schengen, it has caught and spread all legal and technical evolutions set by the European Union to fight illegal migrations. A real testing laboratory for the outsourcing of European migratory policy and of Maghreb cooperation, the Strait of Gibraltar has emerged as a model «frontier-setting» in the Schengen zone, but also as the symbol of its lack of efficiency. Migrants' resourcefulness and capacity to get round European systems and devices underline the total inefficiency of a closing-the-borders policy led without any plan of developing the countries they come from. 
INDEX

Mots-clés : Gibraltar, Schengen, externalisation de la politique migratoire, SIVE, immigration clandestine

Keywords : migratory policy outsourcing, clandestine immigration

\section{AUTEUR}

\section{GUILLAUME LE BOEDEC}

Etudiant, vous pouvez le joindre à l'adresse suivante : gleboedec@hotmail.com 